\title{
2 SAÚDE MENTAL DOS ENFERMEIROS: CONTRIBUTOS DO BURNOUT E ENGAGEMENT NO TRABALHO1
}

\author{
| Sara Faria ${ }^{2}$; Cristina Queirós ${ }^{3}$; Elisabete Borges ${ }^{4}$; Margarida Abreu ${ }^{5} \mid$
}

\section{RESUMO}

INTRODUÇÃO: As novas exigências profissionais e laborais na Enfermagem constituem fatores de risco para a saúde mental dos enfermeiros, cujas consequências individuais podem ser o burnout e a desmotivação no trabalho, bem como, a nível organizacional, a qualidade dos cuidados prestados.

OBJETIVO: Conhecer os níveis de burnout e de engagement numa amostra de enfermeiros, a sua inter-relação e variação em função de características sociodemográficas/laborais.

MÉTODOS: Estudo transversal, descritivo e correlacional, desenvolvido no âmbito do projeto INT-SO "Dos contextos de trabalho à saúde ocupacional dos profissionais de enfermagem". Foram aplicadas versões portuguesas do Maslach Burnout Inventory e da Utrecht Work Engagement Scale, e um questionário de caracterização sociodemográfica/laboral a 346 enfermeiros do distrito do Porto, com participação anónima e voluntária.

RESULTADOS: Encontraram-se níveis elevados de engagement, moderados de exaustão emocional e baixos de despersonalização, existindo $54 \%$ de enfermeiros com nível baixo de burnout, $36 \%$ com moderado e $9 \%$ com elevado. O burnout diminui com a idade e anos de serviço, surge associado a turnos rotativos, a trabalhar em hospitais, tem correlação negativa com o engagement e prediz mais fortemente o engagement (entre 19,6 e 39,6\%) do que o inverso (entre 7,5 e 37,9\%).

CONCLUSÕES: A existência de $9 \%$ de enfermeiros em burnout confirma este grupo como em risco do adoecer psicológico. Os resultados apontam para a importância da saúde mental dos enfermeiros no contexto de trabalho, reforçando a prevenção do burnout e valorizando a saúde mental positiva expressa nas dimensões do engagement dedicação e vigor dos enfermeiros.

PALAVRAS-CHAVE: Saúde mental; Esgotamento profissional; Fenómenos psicológicos; Enfermeiros

\section{RESUMEN}

\section{"Salud mental de enfermeros: Contribuciones de lo burnout y engagement no trabajo"}

INTRODUCCIÓN: Las nuevas demandas profesionales y ocupacionales en enfermería constituyen factores de riesgo para la salud mental de los enfermeros, cuyas consecuencias individuales pueden ser agotamiento y desmotivación en el trabajo, así como, a nivel organizativo, la calidad de los cuidados proporcionados.

OBJETIVO: Conocer los niveles de agotamiento y motivación en una muestra de enfermeras, su interrelación y su variación segundo características sociodemográficas/profesionales.

METODOLOGÍA: Estudio transversal, descriptivo y correlacional, desarrollado bajo el proyecto INT-SO "Desde los contextos de trabajo hasta la salud ocupacional de profesionales de enfermería". Se aplicaron versiones portuguesas de Maslach Burnout Inventory y Utrecht Work Engagement Scale, y un cuestionario de caracterización sociodemográfica/laboral a 346 enfermeros en el distrito de Porto, con participación anónima y voluntaria.

RESULTADOS: Se encontraron altos niveles de motivación, moderado agotamiento emocional y baja despersonalización, con $54 \%$ de enfermeros con nivel bajo de burnout, $36 \%$ con moderado y $9 \%$ con alto. El agotamiento disminuye con la edad y años de servicio, se asocia con turnos rotativos, a trabajar en hospitales, tiene correlación negativa con la motivación y predice más fuertemente la motivación (entre 19,6 y 39,6\%) y non tanto lo inverso (entre $7,5$ y $37,9 \%)$.

CONCLUSIONES: La existencia de $9 \%$ de enfermeros en burnout confirma este grupo como de riesgo para la enfermedad psicológica. Los resultados señalan la importancia de la salud mental de los enfermeros en el contexto de trabajo, fortaleciendo la prevención de burnout y la valoración de la salud mental positiva, expresada en las dimensiones de motivación dedicación y vigor de los enfermeros.

\section{DESCRIPTORES: Salud mental; Agotamiento professional; Fenómenos psicológicos; Enfermeros}

\begin{abstract}
“Nurses' mental health: Contributions of burnout and job engagement"

BACKGROUND: The new professional and labour demands in nursing are risk factors for nurses' mental health, having as individual consequences burnout and job disengagement, as well as, at the organizational level, the quality of care provided.

AIM: To identify the levels of burnout and engagement in a sample of nurses, its interrelationships and its variation according sociodemographic/labour characteristics.

METHODS: A cross-sectional, descriptive and correlational study was developed under the project INT-SO "From work contexts to nursing professionals' occupational health". We applied Portuguese versions of Maslach Burnout Inventory and Utrecht Work Engagement Scale, and a questionnaire to demographic/labour characterization to 346 nurses in Porto district, with anonymous and volunteer participation.

RESULTS: We found high levels of engagement, moderate emotional exhaustion and low depersonalization, having $54 \%$ of nurses a low level of burnout, $36 \%$ a moderate and $9 \%$ a high level. Burnout decreases with age and job experience, it is associated with rotating shifts, working in hospitals, has negative correlation with the engagement, and predicts more strongly engagement (between 19,6 and 39,6\%) than the reverse (between 7,5 and 37,9\%).

CONCLUSIONS: The existence of $9 \%$ of nurses in burnout confirms this group as at risk of the psychologic sickness. The results point to the importance nurses' mental health of in the context of work, strengthening the prevention of burnout and valuing the positive mental health expressed in the dimensions of engagement nurses' dedication and vigour.
\end{abstract}

\section{KEYWORDS: Mental health; Burnout; Psychological phenom- ena; Nurses}

Submetido em 31-03-2019

Aceite em 12-07-2019

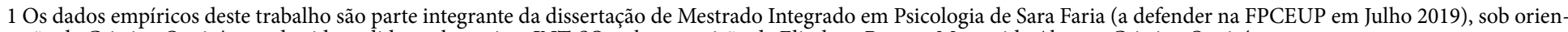
tação de Cristina Queirós, tendo sido cedidos pelo projeto INT-SO, sob supervisão de Elisabete Borges, Margarida Abreu e Cristina Queirós.

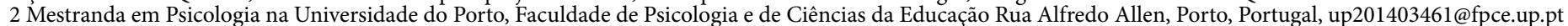

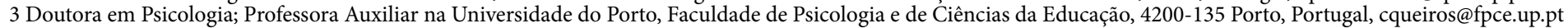

4 Doutora em Enfermagem; Professora Adjunta na Escola Superior de Enfermagem do Porto, 4200-072 Porto, Portugal, elisabete@esenf.pt

5 Doutora em Enfermagem; Professora Coordenadora na Escola Superior de Enfermagem do Porto, 4200-072 Porto, Portugal, mabreu@esenf.pt

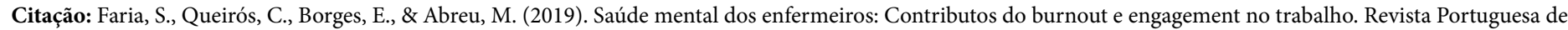
Enfermagem de Saúde Mental (22), 09-18. 


\section{INTRODUÇÃO}

Nos últimos anos a Saúde Mental tem sido alvo de interesse crescente assumindo-se o impacto que a doença mental tem a nível do indivíduo, família, profissão e sociedade e enfrentando por isso novos desafios (Cordeiro, 2018). Em 2017, o Dia Mundial da Saúde Mental elegeu como tema prioritário "Mental health at workplace", reforçando a necessidade de estudos sobre a saúde mental no trabalho, nomeadamente stress no trabalho e burnout, tópicos sobre os quais a Fundação Europeia para a Melhoria das Condições de Vida e de Trabalho (EUROFOUND, 2018) e a Agência Europeia para a Segurança e Saúde no Trabalho (EU-OSHA, 2018) têm vindo a alertar devido aos prejuízos e custos que acarretam na saúde do trabalhador e na produtividade da organização.

Os enfermeiros constituem atualmente um grupo vulnerável ao burnout pois enfrentam no seu trabalho exigências quantitativas, ritmo de trabalho acelerado, sobrecarga de trabalho, sofrimento dos utentes, problemas de colaboração/comunicação em equipa, dificuldade na conciliação trabalho-família, falta de recursos humanos e materiais, entre outros (Hoff, Carabetta \& Collinson, 2019; Marôco et al., 2016).

O burnout foi definido pela psicóloga americana Christine Maslach (Maslach, Schaufeli, \& Leiter, 2001) como uma síndrome de exaustão emocional, despersonalização (ou cinismo) e redução de realização pessoal, que ocorre com frequência nos profissionais que prestam serviços. Inicia-se pela exaustão emocional associada a um sentimento de estar emocionalmente sobrecarregado e exausto no trabalho, culminando num esgotamento dos recursos emocionais do indivíduo. A despersonalização consiste numa resposta insensível e impessoal em relação aos utentes, expressa em atitudes frias e sentimentos negativos e cínicos. Por fim, a redução de realização pessoal refere-se à tendência para se auto avaliar negativamente, particularmente no que diz respeito ao trabalho com outras pessoas, traduzindo-se numa insatisfação consigo mesmo e com as suas concretizações no trabalho. As consequências do burnout podem ser físicas, psicológicas e ocupacionais, abrangendo vertentes importantes na vida do indivíduo e levando até mesmo ao suicídio, enquanto em termos organizacionais aumentam o turnover, absentismo, presentismo e insatisfação no trabalho, bem como prejudicam a qualidade dos cuidados prestados (EUROFOUND, 2018; Marques-Pinto et al., 2015, 2018; Maslach et al., 2001; Salvagioni et al., 2017).
Estudos recentes apontam para a ligação entre burnout e saúde mental alertando para a necessidade de desenvolver estratégias que desde o início previnam o burnout, seus perfis clínicos e associação a outras doenças mentais como depressão e ansiedade, bem como reconheçam o papel dos enfermeiros no sistema de saúde (Johnson et al., 2018; Koutsimani, Montgomery \& Georganta, 2019).

Tentando contrariar esta tendência negativa e de sofrimento provocado pelo trabalho, alguns autores optaram por abordagens de saúde mental positiva (Barry, 2009; Lluch Canut et al., 2017), na qual o conceito de engagement pode ser enquadrado enquanto fator promotor (Watanabe \& Yamauchi, 2018) pois apesar de todas as exigências referidas, os enfermeiros, estão motivados e empenhados em realizar bem a sua tarefa (Cunha et al., 2018).

O engagement surge com o aumento do interesse pela Psicologia Positiva em detrimento do foco no tratamento de doenças mentais, procurando o bem-estar mental do profissional (Schaufeli \& De Witte, 2017). Pode ser definido como um estado mental positivo e satisfatório, relacionado com o trabalho e que é caracterizado pelo vigor, dedicação e absorção. $\mathrm{O}$ vigor traduz-se em altos níveis de energia e resiliência mental durante o trabalho, disposição para investir esforços e persistir no trabalho mesmo perante dificuldades. A dedicação implica orgulho no seu trabalho e investimento, enquanto a absorção implica concentração e envolvimento nas tarefas. Existe uma discussão se é o oposto do burnout num contínuo de estado mental associado ao trabalho pois relaciona-se negativamente com este, mas como não apresenta correlações quase completas e associação a outras variáveis, pode ser entendido como um construto independente do burnout (Schaufeli \& De Witte, 2017).

Vários estudos referem o engagement como fator protetor do burnout, demonstrando que os trabalhadores com elevado engagement, por oposição aos que apresentam elevado burnout, têm maior capacidade para gerir as suas necessidades diárias e trabalhar com felicidade (Bodine, 2018; Watanabe \& Yamauchi, 2018). Além disso, níveis mais altos de engagement nos enfermeiros e índices de pessoal mais favoráveis entre enfermeiro/utente estão associados a percepções positivas de segurança do paciente, constituindo uma mais valia não apenas para o enfermeiro como também para os pacientes e instituição de trabalho (Brooks-Carthon et al., 2018). 
Melhorar o engagement e a equipa de trabalho do enfermeiro também traz benefícios importantes para a força de trabalho, uma vez que os enfermeiros a exercer funções em ambientes exemplares de prática profissional relatam menos burnout, rotatividade e turnover, bem como melhor qualidade na interação e envolvimento com o utente (Kutney-Lee et al., 2016; MarquesPinto et al., 2015, 2018). Contudo, nem sempre existe reconhecimento do desgaste que o cuidado concedido pelos enfermeiros pode ter na sua saúde, uma vez que o enfoque assenta somente na maximização da vida dos outros e qualidade eficiente que são oferecidos por estes profissionais à população onde se inserem, o que poderá levar a uma redução no alerta e prevenção do seu bem estar e saúde ocupacional.

Este estudo tem como objetivo conhecer os níveis de burnout e de engagement numa amostra de enfermeiros, do distrito do Porto, a inter-relação entre burnout e engagement (nomeadamente como preditores recíprocos) e a sua variação em função de características sociodemográficas e laborais.

\section{MÉTODOS}

\section{Participantes}

Estudo transversal, descritivo e correlacional, desenvolvido no âmbito do projeto INT-SO "Dos contextos de trabalho à saúde ocupacional dos profissionais de enfermagem" com recurso a amostras de conveniência e amostras em bola de neve em função dos estudos em curso no projeto (sem diferenças nos resultados em função do tipo de amostra), num total de 346 enfermeiros do distrito do Porto.

\section{Instrumentos}

Foram aplicados o Maslach Burnout Inventory (MBI, desenvolvido por Maslach e colaboradores na década de 90), a Utrecht Work Engagement Scale (UWES, construído por Schaufeli e Bakker a partir de 2000) e um questionário de caracterização sociodemográfica/ laboral, o qual inquiriu sobre a idade, sexo, estado civil, existência de filhos, habilitações, tipo de turno, vínculo de trabalho e anos de experiência profissional.

O Maslach Burnout Inventory é composto por 22 itens avaliados numa escala de 7 pontos (varia de $0=$ nunca até $6=$ todos os dias) e organizados em três dimensões: exaustão emocional, despersonalização e realização pessoal. A exaustão emocional inclui 9 itens que avaliam a falta de recursos emocionais, sentimentos de sobrecarga emocional e exaustão causada pelo trabalho, enquanto a despersonalização tem 5 itens que avaliam a insensibilidade e indiferença em relação aos utentes a quem se presta serviço. Por fim, a realização pessoal inclui 8 itens que avaliam os sentimentos de realização e competência no trabalho.

É calculada a média por dimensão, significando um valor mais elevado maior nível na dimensão, podendo ser calculada a média da escala global (burnout) desde que sejam invertidos os itens da dimensão realização pessoal e recalculada a média dos 22 itens.

A Utrecht Work Engagement Scale é composta por 17 itens avaliados numa escala de 7 pontos (varia de $0=$ nunca até $6=$ todos os dias) e organizados em três dimensões: vigor, dedicação e absorção.

$O$ vigor inclui 6 itens que avaliam a energia, entusiasmo e persistência perante dificuldades no trabalho.

A dedicação é composta por 5 itens que avaliam o significado atribuído ao trabalho e o orgulho e investimento no trabalho.

A absorção inclui 6 itens e avalia a concentração e imersão no trabalho sem sentir o tempo a passar, bem como a dificuldade em se separar do trabalho devido ao entusiasmo e investimento. Note-se que em determinadas profissões esta dimensão apresenta valores inferiores devido às características da tarefa que não se propiciam ao estado de concentração mental. É ainda possivel calcular o valor global da escala (engagement) através da média dos 17 itens.

Atendendo á proliferação de traduções do MBI e do UWES, optou-se por recorrer às versões portuguesas utilizadas no projeto europeu RN4Cast sobre a influência de variaveis organizacionais no recrutamento e retenção de enfermeiros e que em Portugal recolheu dados de 2.235 participantes (respetivamente Jesus et al., 2014; Marques-Pinto et al., 2015), com boas qualidades psicométricas.

Note-se que estas versões portuguesas têm sido amplamente utilizadas noutros estudos (como por exemplo nos citados por Marques-Pinto \& Chambel, 2008) desde a sua adaptação inicial por Marques-Pinto no início de 2000 e com validação posterior por Chambel e Oliveira-Cruz (2008), os quais efetuaram análises fatoriais confirmatórias através do método de equações estruturais para um modelo de bem-estar, tendo nos diferentes estudos encontrado (Tabela 1) alfas de Cronbach entre 0,59 e 0,89 enquanto com enfermeiros, no projeto RN4Cast foram encontrados alfas entre 0,72 e 0,87 para as dimensõees do burnout (Jesus et al., 2014) e de 0,94 para o engagement total (Marques-Pinto et al., 2015). 
À semelhança de outras amostras portuguesas, na presente amostra (Tabela 1) foram encontradas adequadas qualidades psicométricas (Field, 2009), embora a despersonalização e a absorção apresentem um alfa ligeiramente inferior, pois caracterizando-se pela frieza emocional na interação com os utentes e pelo "deixar ir" na tarefa (típica de tarefas de elevada concentração mental), não parecem ser, respetivamente, habituais nos enfermeiros nem tão frequentes no seu protocolo de atuação. Conforme os valores de alfa habitualmente apresentados (dimensões e escala global, respetivamente burnout e engagement), os valores de alfa nesta amostra seguem, então, a tendência de outros estudos com amostras portuguesas. Note-se que para todas as variaveis estudadas, o erro padrão da assimetria foi de 0,131 e o da curtose foi de 0,261 enquanto o teste de Kolmogorov-Smirnov foi significativo. Assim, não estando cumpridos todos os pressupostos de normalidade, optou-se nas análises comparativas por utilizar testes não paramétricos.

Tabela 1 - Características psicométricas do MBI e UWES

\begin{tabular}{|l|c|c|c|c|c|c|}
\hline \multirow{2}{*}{ Dimensões } & \multicolumn{3}{|c|}{ Alfa Cronbach } & & \\
\cline { 2 - 7 } & $\begin{array}{c}\text { Chambel \& Oliveira-Cruz } \\
(2008)\end{array}$ & RN4Cast & $\begin{array}{c}\text { Presente } \\
\text { estudo }\end{array}$ & $\begin{array}{c}\text { Assimetria } \\
\text { Curtose }\end{array}$ & $\begin{array}{c}\text { Kolmogorov-Smirnov } \\
(\mathrm{p})\end{array}$ \\
\hline Exaustão Emocional & 0,82 a 0,83 & $0,87 \mathrm{a}$ &, 880 &, 118 &,- 730 &, $069\left(, 000^{* * *}\right)$ \\
\hline Despersonalização & 0,59 a 0,70 & $0,72 \mathrm{a}$ &, 751 & 1,383 &, $167\left(, 000^{* * *}\right)$ \\
\hline Realiz. Pessoal & 0,76 a 0,82 & $0,79 \mathrm{a}$ &, 784 &,- 803 &, 778 &, $109\left(, 000^{* * *}\right)$ \\
\hline Burnout & - & - &, 870 &, 265 &,- 391 &, $050\left(, 035^{* * *}\right)$ \\
\hline Vigor & 0,82 a 0,89 & - &, 880 & $-1,176$ &, $233\left(, 000^{* * *}\right)$ \\
\hline Dedicação & 0,87 a 0,89 & - &, 906 & $-1,109$ &, 532 &, $224\left(, 000^{* * *}\right)$ \\
\hline Absorção & 0,59 a 0,67 & - &, 695 &,- 888 &, 243 &, $141\left(, 000^{* * *}\right)$ \\
\hline Engagement & - & $0,94 b$ &, 929 & $-1,127$ &, 697 &, $166\left(, 000^{* * *}\right)$ \\
\hline
\end{tabular}

\section{PROCEDIMENTOS}

O estudo decorreu no âmbito do projeto INT-SO “Dos contextos de trabalho à saúde ocupacional dos profissionais de enfermagem", desenvolvido na Escola Superior de Enfermagem do Porto e aprovado pela ESEP (Comissão de Ética, anexo à ata n. $\left.{ }^{\circ} 8 / 2016\right)$. Em função dos estudos inseridos neste projeto, foram utilizadas amostras de conveniência e amostras em bola de neve, o que, por um lado aumenta exponencialmente a divulgação do estudo, mas por outro impede de identificar a amplitude de possiveis participantes e a taxa de adesão. Assim, as amostras de conveniência correspondem a dissertações de mestrado que integravam o INT-SO, tendo sido entregues questionários em papel a enfermeiros que integravam instituições contactadas previamente, e que como à data não tinham Comissão de Ética, autorizaram a recolha desde que fosse com participação voluntária dos seus trabalhadores. As amostras em bola de neve partiram de uma lista inicial de 50 contactos de enfermeiros a realizar formação pós-graduada na ESEP, que divulgaram, através de um link para os questionários online, o estudo junto de colegas das respetivas instituições, convidando-os à participação.Todas as participações foram voluntárias e anónimas, de forma a respeitar os requisitos formais e éticos necessários à investigação.
Os dados recolhidos foram processados no IBM SPSS Statistics (versão 25), recorrendo a testes de normalidade, análises descritivas, teste não paramétrico de Mann-Whitney para amostras independentes, Coeficiente Correlação R de Pearson e Regressão métodos Enter e Stepwise, de acordo com recomendações de Field (2009).

\section{RESULTADOS}

A amostra apresentou idades entre os 22 e os 60 anos (média de 34,5 e desvio padrão de 8,4 ), sendo $72 \%$ do sexo feminino, $50 \%$ casados ou em união de facto, $43 \%$ com filhos e $61 \%$ com bacharelato/licenciatura (restantes com pós-graduação ou mestrado). A maioria dos participantes (67\%) trabalhava em hospitais ou centros de saúde (25\%) e $8 \%$ em lares ou clínicas, $66 \%$ trabalhava por turnos rotativos (restantes em turnos fixos), $68 \%$ tinha vínculo definitivo (restantes vínculo precário), tendo em média 11,6 anos de experiência profissional (desvio padrão de 8,3). A análise dos níveis de burnout, utilizando as categorias definidas por Marôco e colaboradores (2016) revelou (Tabela 2) que $54 \%$ da amostra apresenta nível baixo de burnout, 36\% nível moderado e 9\% nível elevado, enquanto para o engagement, $7 \%$ da amostra apresentava um nível baixo, $8 \%$ nível moderado e $85 \%$ nível elevado. 
Tabela 2 - Percentagem por nível de burnout e engagement

\begin{tabular}{|c|c|c|}
\hline Níveis & Burnout (\%) & Engagement (\%) \\
\hline Baixo & 54,3 & 6,6 \\
\hline Médio & 36,4 & 8,4 \\
\hline Elevado & 9,3 & 85,0 \\
\hline
\end{tabular}

A análise descritiva por dimensão revelou, para o burnout, níveis moderados de exaustão emocional, baixos de despersonalização, elevados de realização pessoal e elevados para todas as dimensões do engagement, tendo a absorção os valores mais baixos (Tabela 3). A análise correlacional demonstrou que o burnout e a despersonalização diminuem com a idade e anos de experiência profissional, diminuindo a exaustão emocional à medida que aumentam os anos de experiência profissional. O engagement não apresenta esta variação, apresentando apenas correlação negativa com todas as dimensões do burnout, de menor intensidade para a absorção.

Tabela 3 - Média, desvio padrão e correlações de Pearson entre idade, experiência profissional, burnout e engagement

\begin{tabular}{|c|c|c|c|c|c|c|c|c|}
\hline Dimensões (0-6) & Média & DP & Idade & $\begin{array}{l}\text { Experiência } \\
\text { Profissional }\end{array}$ & E. Emoc & Desp. & Realiz. P & Burnout \\
\hline Exaustão Emocional & 2,728 & 1,276 &,- 112 &,$- 146^{\star *}$ & & & & \\
\hline Despersonalização & 1,069 & 1,105 &,$- 150^{\star *}$ &,$- 197^{\star *}$ & & & & \\
\hline Realização Pessoal & 4,512 & 861 & ,056 & 087 & & & & \\
\hline Burnout & 1,901 & 822 &,$- 137^{\star}$ &,$- 186^{\star *}$ & & & & \\
\hline Vigor & 4,370 & 1,316 &, 089 &, 096 &,$- 496^{* *}$ &,$- 228^{\star *}$ &, $498^{\star *}$ &,$- 575^{\star *}$ \\
\hline Dedicação & 4,439 & 1,406 & ,079 &, 085 &,$- 497^{\star *}$ &,$- 247^{\star \star}$ &, $496^{\star *}$ &,$- 581^{\star *}$ \\
\hline Absorção & 4,166 & 1,364 & ,077 & ,077 &,$- 361^{\star *}$ &,$- 148^{\star \star}$ &, $339 * *$ &,$- 404^{\star *}$ \\
\hline Engagement & 4,324 & 1,255 & 087 & ,091 &,$- 490^{\star *}$ &,$- 227^{\star *}$ & $482^{\star *}$ &,$- 565^{\star *}$ \\
\hline
\end{tabular}

${ }^{*} \mathrm{p} \leq, 050 \quad{ }^{* *} \mathrm{p} \leq, 010 \quad{ }^{* * *} \mathrm{p} \leq, 001 \quad$ a Jesus et al. (2014) $\quad$ b Marques-Pinto et al. (2015)

A análise comparativa em função das variáveis sociodemográficas e laborais não revelou diferenças estatisticamente significativas para o sexo, estado civil, existência de filhos, e habilitações. Para o vínculo de trabalho (Tabela 4) encontrou-se mais realização pessoal associada ao vínculo precário. Já no que se refere ao turno, embora com valores semelhantes na realização pessoal, encontrou-se mais exaustão emocional, despersonalização e burnout nos enfermeiros com turno rotativo, bem como menos vigor, dedicação, absorção e engagement. Relativamente ao local de trabalho os hospitais apresentam valores superiores na exuastão emocional, despersinalização e burnout, enquanto a realização pessoal e todas as dimensões do engagement são superiores nos centros de saúde. Dado o valor residual de enfermeiros a exercer noutros locais de trabalho (8\%) não foram incluídos nesta comparação.

Tabela 4 - Apresentação de médias e análise comparativa não paramétrica em função do turno e local de trabalho

\begin{tabular}{|l|c|c|c|c|c|}
\hline Dimensões (0-6) & $\begin{array}{c}\text { Vinculo definitivo } \\
(\mathbf{N = 2 3 6 )}\end{array}$ & $\begin{array}{c}\text { Vinculo precário } \\
\mathbf{( N = 1 0 4 )}\end{array}$ & $\begin{array}{c}\text { Mean Rank v. } \\
\text { definitivo }\end{array}$ & $\begin{array}{c}\text { Mean Rank v. } \\
\text { precário }\end{array}$ & P Mann-Whitney \\
\hline Realização Pessoal & 4,47 & 4,64 & 163,05 & 187,40 &, $035^{\star}$ \\
\hline Exaustão Emocional & $\begin{array}{c}\text { Turno fixo } \\
(\mathbf{N = 1 1 4 )}\end{array}$ & $\begin{array}{c}\text { Turno rotativo } \\
(\mathbf{N}=\mathbf{2 2 3})\end{array}$ & Mean Rank t. fixo & $\begin{array}{c}\text { Mean Rank t. } \\
\text { rotativo }\end{array}$ & \\
\hline Despersonalização & 2,38 & 2,88 & 144,85 & 181,35 &, $001^{* * \star}$ \\
\hline Burnout & 0,71 & 1,24 & 137,34 & 185,19 &, $000^{* * *}$ \\
\hline Vigor & 1,64 & 2,01 & 140,07 & 183,79 &, $000^{* * *}$ \\
\hline Dedicação & 4,60 & 4,27 & 183,82 & 161,43 &, $042^{\star}$ \\
\hline Absorção & 4,70 & 4,31 & 184,57 & 161,04 &, $035^{\star}$ \\
\hline Engagement & 4,41 & 4,05 & 184,43 & 161,11 &, $037^{\star}$ \\
\hline
\end{tabular}




\begin{tabular}{|l|c|c|c|c|c|}
\hline & $\begin{array}{c}\text { Hospital } \\
(\mathbf{N = 2 3 3 )}\end{array}$ & $\begin{array}{c}\text { Centro de Saúde } \\
(\mathbf{N = 8 5})\end{array}$ & $\begin{array}{c}\text { Mean Rank } \\
\text { hospital }\end{array}$ & $\begin{array}{c}\text { Mean Rank } \\
\text { c. Saúde }\end{array}$ & \\
\hline Exaustão Emocional & 2,84 & 2,41 & 167,82 & 136,69 &, $008^{* *}$ \\
\hline Despersonalização & 1,18 & 0,73 & 170,06 & 130,54 &, $001^{* * *}$ \\
\hline Realização Pessoal & 4,46 & 4,66 & 153,26 & 176,82 &, $045^{\star}$ \\
\hline Burnout & 1,99 & 1,64 & 170,34 & 129,78 &, $000^{* * *}$ \\
\hline Vigor & 4,29 & 4,76 & 149,81 & 186,06 &, $002^{* *}$ \\
\hline Dedicação & 4,34 & 4,87 & 150,27 & 184,80 &, $003^{* *}$ \\
\hline Absorção & 4,02 & 4,70 & 146,30 & 195,68 &, $000^{* * *}$ \\
\hline Engagement & 4,22 & 4,77 & 147,06 & 193,59 &, $000^{* * *}$ \\
\hline
\end{tabular}

${ }^{*} \mathrm{p} \leq, 050 \quad{ }^{* *} \mathrm{p} \leq, 010 \quad{ }^{* * *} \mathrm{p} \leq, 001$

Através da análise de regressão método Enter (Tabela 5) verificou-se que o burnout e o engagement são preditores recíprocos, ambos sofrendo a influência de variáveis sociodemográficas (habilitações académicas, sexo, estado civil, idade e existência de filhos) e laborais (vínculo, turno, anos de experiência e antiguidade na instituição). Contudo, o burnout prediz mais fortemente as dimensões do engagement (entre 19,6 e 39,6\%) do que o engagement prediz o burnout (entre 7,5 e 37,9\%). Verificou-se ainda uma influência residual das variáveis sociodemográficas na exaustão emocional, mas cuja significância desaparece numa análise pelo método Stepwise, o mesmo acontecendo na influência das variáveis laborais no burnout e engagement, exceto na influência dos turnos rotativos na maior despersonalização $(p=, 050)$ e do sexo feminino na maior dedicação $(p=, 003)$.

Tabela 5 - Variáveis preditoras do burnout e do engagement (regressão método Enter)

\begin{tabular}{|c|c|c|c|c|c|}
\hline Dimensões & Preditores & R Square & R Square change & $\mathbf{F}$ & $\mathbf{p}$ \\
\hline \multirow[t]{3}{*}{ Exaustão Emocional } & Engagement & 268 & ,268 & 38,049 &, $000^{\star * *}$ \\
\hline & Laborais & 296 & 027 & 2,373 & $039^{*}$ \\
\hline & Sociodemográficas & 318 & 022 & 2,432 &, $048^{\star}$ \\
\hline \multirow[t]{3}{*}{ Despersonalização } & Engagement & 075 & 075 & 8,394 &, $000^{\star \star \star}$ \\
\hline & Laborais & , 129 & 054 & 3,800 &, $002^{\star \star}$ \\
\hline & Sociodemográficas & , 138 & ,009 & 820 & ,513 \\
\hline \multirow[t]{3}{*}{ Realização Pessoal } & Engagement & 295 & 295 & 43,472 &, $000^{* * *}$ \\
\hline & Laborais & ,298 & 003 & ,242 & ,944 \\
\hline & Sociodemográficas & ,308 &, 010 & 1,074 & 370 \\
\hline \multirow[t]{3}{*}{ Burnout } & Engagement & 379 & 379 & 63,375 &, $000^{\star * *}$ \\
\hline & Laborais & 415 & 036 & 3,749 &, $003^{\star *}$ \\
\hline & Sociodemográficas & ,425 &, 010 & 1,277 & 279 \\
\hline \multirow[t]{3}{*}{ Vigor } & Burnout & ,396 & ,396 & 67,878 &, $000^{\star * \star}$ \\
\hline & Laborais & ,404 & 009 & 884 & ,492 \\
\hline & Sociodemográficas & ,417 &, 013 & 1,688 &, 153 \\
\hline \multirow[t]{3}{*}{ Dedicação } & Burnout & ,393 & ,393 & 67,181 &, $000^{\star \star \star}$ \\
\hline & Laborais & 402 & ,009 & 887 & 490 \\
\hline & Sociodemográficas & ,420 & 019 & 2,413 &, $049^{*}$ \\
\hline \multirow[t]{3}{*}{ Absorção } & Burnout & , 196 & , 196 & 25,243 &, $000^{\star * \star}$ \\
\hline & Laborais & 204 & ,008 & 592 & ,706 \\
\hline & Sociodemográficas & 210 & 007 & 629 & ,642 \\
\hline \multirow[t]{3}{*}{ Engagement } & Burnout & ,376 & ,376 & 62,465 &, $000^{* * *}$ \\
\hline & Laborais & ,383 & 007 & 684 & ,636 \\
\hline & Sociodemográficas & ,396 & 013 & 1,598 & 175 \\
\hline
\end{tabular}

${ }^{*} \mathrm{p} \leq, 050 \quad{ }^{* *} \mathrm{p} \leq, 010 \quad{ }^{* * *} \mathrm{p} \leq, 001$ 


\section{DISCUSSÃO}

Apesar de se terem encontrado valores moderados de exaustão emocional e baixos de despersonalização, existem já 36\% de enfermeiros com nível moderado e $9 \%$ com nível elevado de burnout, confirmando este grupo profissional como em risco do adoecer psicológico, tal como encontrado por outros estudos (Hoft et al., 2019; Salvagioni et al., 2017). Note-se que estes valores são diferentes dos encontrados a nível nacional por Marôco e colaboradores (2016), com $21 \%$ de enfermeiros em burnout moderado, mas $49 \%$ em burnout elevado. Também Jesus e colaboradores (2014) alertaram que os enfermeiros têm vindo a apresentar níveis crescentes de exaustão emocional, o que deve constituir uma preocupação nos gestores das organizações. É de alertar que os valores do presente estudo podem estar abaixo da realidade, pois foi utilizada uma amostra de voluntários, e pelo mito do trabalhador saudável os trabalhadores em pior estado psicológico já não têm capacidade de participar em estudos, gastando as suas energias em enfrentar os stressores laborais (Shah, 2009).

Os valores de engagement encontrados são elevados, consolidando a relação entre estes dois conceitos (Schaufeli \& De Witte, 2017) e estando concordantes com a literatura que refere a elevada dedicação e motivação dos enfermeiros, seja em estudos nacionais (Cunha et al., 2018; Marques-Pinto et al., 2015, 2018) ou internacionais (Watanabe \& Yamauchi, 2018).

O facto de maior despersonalização e burnout surgirem associados a mais idade e experiência profissional, e de a exaustão emocional se associar apenas à experiência profissional, enquanto a realização pessoal não apresenta associações significativas, é coerente com um outro estudo sobre fatores de risco nos enfermeiros e alerta para o efeito cumulativo da sobrecarga emocional ao longo dos anos, qualquer que seja a idade, sugerindo a influência de variáveis organizacionais ou laborais e não vulnerabilidade do profissional (Hoff et al., 2019). Esta ideia é reforçada pelas análises comparativas que não revelaram existência de diferenças em função de variáveis individuais, mas revelaram que os turnos rotativos e trabalhar num hospital estão associados a maior nível de burnout, resultados encontrados noutros estudos (Jesus et al., 2014; Johnson et al., 2018).

$\mathrm{O}$ facto de o vinculo precário surgir associado a mais realização pessoal pode justificar-se por salários mais elevados ao acumular tarefas em diferentes instituições (Marques-Pinto \& Chambel, 2008), bem como ao facto de, talvez pela crise socioeconómica que forçou muitos enfermeiros portugueses a emigrar, os que ficaram se sentirem realizados por terem um vinculo institucional ou não vivenciarem tanto com os conflitos hierárquicos da instituição de quem está já com um vinculo definitivo (Gillet et al., 2019), resultado também encontrado por Dutra e colaboradores (2019).

É de realçar que no presente estudo não foram encontradas diferenças em função do sexo frequentemente referidas, talvez pela maioria do sexo feminino $(72 \%)$, o que poderá ter abafado resultados diferenciais, já que outros estudos (Jesus et al., 2014; Marques-Pinto et al., 2018) e até uma meta-análise (Purvanova \& Muros, 2010) revelaram que o sexo feminino apresenta maiores níveis de exaustão emocional e o sexo masculino mais despersonalização, o que remete para estratégias de intervenção ou prevenção diferenciadas para cada sexo.

Além disso, o facto de o burnout explicar de forma mais elevada o engagement do que o inverso, revela a nocividade deste, minando a capacidade do profissional se envolver no seu trabalho e daí retirar prazer. Assim, os impactos individuais e laborais do burnout destacam a necessidade de intervenções preventivas e da identificação precoce desta condição de saúde mental no ambiente de trabalho (Bodine, 2018; Salvagioni et al, 2017). Note-se que no estudo português do projeto RN4CAST foi demonstrado o elevado burnout mas também elevado engagement de enfermeiros (Jesus et al., 2014; Marques-Pinto et al., 2015), bem como o efeito mediador de ambos na intenção de mudar de instituição, sugerindo-se a redução dos sintomas de exaustão emocional e a promoção do engagement para reter enfermeiros qualificados pois encontraram $43 \%$ de enfermeiros a manifestar intenção de mudar de instituição. Apesar dos inúmeros estudos sobre burnout e engagement em enfermeiros, sugere-se no futuro analisar outras variáveis como satisfação no trabalho, interação trabalho-família, etc., pois contribuem para a interação burnout-engagement, constituindo fatores protetores ou de vulnerabilidade, pois constitui uma limitação deste estudo ter sido centrado apenas no burnout e engagement.

Outras limitações resultam de terem sido utilizadas amostras constituídas por voluntários, não podendo os resultados ser generalizáveis por a amostra total do estudo não poder ser considerada como representativa dos dados nacionais em características profissionais nem em número. 


\section{CONCLUSÕES}

Constituindo os enfermeiros a maioria dos profissionais de saúde e vivenciando a cultura hospitalar de forma muito específica devido às suas tarefas de constante interação com os utentes e outros profissionais das instituições de saúde, importa refletir sobre a existência de $9 \%$ da amostra com níveis de burnout elevados.

Assim, interessa reforçar a prevenção do burnout através da inteligência emocional, do engagement e do bem-estar no trabalho, tendo como objetivo melhorar a saúde mental e a saúde ocupacional dos enfermeiros. Nesta amostra, 7\% apresenta já engagement baixo, sugerindo um processo de adoecer mental que com o tempo tem tendência a agravar-se nos próprios enfermeiros já a vivenciar mal-estar psicológico, e a longo prazo, prejudica a instituição de saúde onde exercem pois poderão cometer erros ou afastar-se por bixa médica, sobrecarregando os restantes colegas e assim criando um círculo vicioso de burnout contagioso na equipa. Contudo, se for potenciado o engagement (que nesta amostra é elevado para $85 \%$ dos enfermeiros), sabe-se que trabalhadores com altos níveis de engagement, em oposição aos de elevado burnout, têm mais capacidades para enfrentar melhor as dificuldades do trabalho, as quais são muitas no contexto atual da Enfermagem.

O burnout tem sido considerado um problema de saúde ocupacional e da organização, por vezes esperando-se que esta modifique o seu funcionamento, o que a acontecer, é complexo e moroso.

Independentemente de alguns autores sugerirem que o burnout é uma forma específica de depressão relacionada com o mundo do trabalho, recentemente foi demonstrado que é um constructo independente, mas com ligações com a depressão e ansiedade. Urge, pois, que os serviços de saúde ocupacional se debrucem sobre este fenómeno psicológico e que através do engagement contribua para o novo paradigma da saúde mental positiva.

\section{IMPLICAÇÕES PARA A PRÁTICA CLÍNICA}

Este estudo pretende alertar para o sofrimento mental e psicológico que os enfermeiros podem vivenciar no seu trabalho, apesar de toda a dedicação e envolvimento que demonstram nas suas tarefas.

O facto de o burnout se associar a outras doenças mentais e ter como consequência a diminuição da qualidade dos serviços prestados aos utentes, deve alertar os gestores das instituições de saúde para estarem atentos ao bem-estar dos seus profissionais, estimulando a promoção da saúde mental através da Enfermagem do Trabalho ou de Serviços de Saúde Ocupacional.

Nem sempre existe reconhecimento do desgaste que o cuidado concedido pelos enfermeiros pode ter na sua saúde, pois o enfoque assenta na maximização da vida dos outros e nos cuidados de qualidade oferecidos por estes profissionais aos utentes, o que poderá levar a uma redução no alerta e prevenção do seu próprio bem-estar, saúde mental e saúde ocupacional. Neste sentido, a procura pelo bem- estar e saúde dos enfermeiros deverá incluir intervenções para melhorar a saúde psicológica, ajudar a encontrar a felicidade no local de trabalho e valorizar o engagement no trabalho, em vez de tentar reduzir apenas os fatores de stress e causas de burnout. Contudo, os enfermeiros também poderão ter um papel ativo no sentido de cuidarem de si e estarem atentos aos sintomas negativos resultantes do stress no trabalho crónico, bem como tomarem consciência das implicações que o burnout pode acarretar na sua prática clínica e na sua saúde mental e física.

Atendendo às exigências da vida atual e do modelo de ensino pós-Bolonha, sugere-se que as Escolas de Enfermagem, no âmbito dos seus planos de estudo, incluam conteúdos relacionados com a gestão do stress e prevenção do burnout (até porque este tem vindo a revelar-se uma preocupação pelo seu aumento nos estudantes das áreas da saúde), bem como de estratégias de motivação para a tarefa (engagement) e de reforço da resiliência individual. Além disso, a investigação em Enfermagem, que tem vindo a estudar o burnout, pode contribuir para o desenvolvimento, implementação e análise do impacto de programas de gestão do stress e de prevenção do burnout ou de promoção do engagement e da saúde mental.

Assim, a adoção do paradigma da saúde mental positiva, reforçando estratégias promotoras do engagement, da partilha, do suporte mútuo e do reconhecimento do esforço e dedicação dos enfermeiros pode contribuir para reverter o aumento exponencial do burnout na sociedade atual e para as instituições de saúde terem enfermeiros, que, para além da sua elevada dedicação, estejam motivados e satisfeitos com a sua atividade profissional. 


\section{REFERÊNCIAS BIBLIOGRÁFICAS}

Barry, M. M. (2009). Addressing the determinants of positive mental health: Concepts, evidence and practice. International Journal of Mental Health Promotion, 11(3), 4-17. Doi: 10.1080/14623730.2009.9721788

Bodine, J. L. (2018). Preventing preceptor burnout through engagement. Journal for Nurses in Professional Development, 34(5), 290-292. Doi:10.1097/ nnd. 0000000000000473

Brooks-Carthon, J. M., Hatfield, L., Plover, C., Dierkes, A., Davis, L., Hedgeland, T., \& Aiken, L. H. (2018). Association of Nurse Engagement and Nurse Staffing on Patient Safety. Journal of Nursing Care Quality, 34(1), 1. Doi: 10.1097/NCQ.0000000000000334

Chambel, M.J. \& Oliveira-Cruz, F. (2008). A rutura do contrato psicológico e o desenvolvimento do burnout e do engagement. In A. Marques-Pinto \& M.J. Chambel (Eds.). Burnout e engagement em contexto organizacional: Estudos com amostras portuguesas (pp.145-166). Lisboa: Livros Horizonte.

Cordeiro, R. (2018). Os novos desafios para a saúde mental na Europa. Revista Portuguesa de Enfermagem de Saúde Mental, 20, 6-8. Doi: 10.19131/rpesm.0220

Cunha, S., Gama, C., Fevereiro, M., Vasconcelos, A., Sousa, S., Neves, A., Casanova, J., Teixeira, M., Rodrigues, S., Ribeira, S., \& Firmino-Machado, J. (2018). A felicidade e o engagement no trabalho nos cuidados de saúde primários. Revista Portuguesa de Medicina Geral e Familiar, 34(1), 26-32. Disponível em: http:// www.scielo.mec.pt/scielo.php?script=sci_arttext\&pid $=\mathrm{S} 2182-51732018000100004$

Dutra H.S., Gomes, P.A.L., Garcia, R.N., Oliveira, H.C., Freitas, S.C., \& Guirardello, E.B. (2019). Burnout entre profissionais de enfermagem em hospitais no Brasil. Revista Cuidarte,10(1), e585. Doi.: 10.15649/cuidarte. v10i1.585

EU-OSHA (2018). Healthy workers, thriving companies - a practical guide to wellbeing at work. Luxembourg: Publications Office of the European Union.
EUROFOUND (2018). Burnout in the workplace: A review of data and policy responses in the EU. Luxembourg: Publications Office of the European Union.

Field, A. (2009). Descobrindo a estatística usando o SPSS. (2 ${ }^{\mathrm{a}}$ ed.). Porto Alegre: Artmed Editora.

Gillet, N., Le Gouge, A., Pierre, R., Bongro, J., Méplaux, V., Brunault, P., ... Cheyroux, P. (2019). Managerial style and well-being among psychiatric nurses: A prospective study. Journal of Psychiatric and Mental Health Nursing. Doi:10.1111/jpm.12544

Hoff, T., Carabetta, S., \& Collinson, G.E. (2019). Satisfaction, burnout, and turnover among nurse practitioners and physician assistants: A review of the empirical literature. Medical Care Research and Review, 76(1), 3-31. Doi: 10.1177/1077558717730157

Jesus E. H., Marques-Pinto A., Fronteira I. S. E., \& Mendes A. M. O. C. (2014). Estudo RN4Cast em Portugal: Percepção dos enfermeiros sobre burnout. Revista Investigação em Enfermagem, 9(2), 47-59. Disponível em: https://www.rcaap.pt/detail.jsp?id=oai:repositorio. esenfc.pt:4863

Johnson, J., Hall, L. H., Berzins, K., Baker, J., Melling, K., \& Thompson, C. (2018). Mental healthcare staff well-being and burnout: A narrative review of trends, causes, implications, and recommendations for future interventions. International Journal of Mental Health Nursing, 27(1), 20-32. Doi:10.1111/inm.12416

Koutsimani, P., Montgomery, A., \& Georganta, K. (2019). The relationship between burnout, depression, and anxiety: A systematic review and meta-analysis. Frontiers in Psychology, 10, 284. Doi: 10.3389/ fpsyg.2019.00284

Kutney-Lee, A., Germack, H., Hatfield, L., Kelly, S., Maguire, P., Dierkes, A., \& Aiken, L. H. (2016). Nurse Engagement in Shared Governance and Patient and Nurse Outcomes. Journal of Nursing Administration, 46(11), 605-612. Doi: 0.1097/NNA.0000000000000412

Lluch Canut, M. T., Sequeira, C., \& Roldán-Merino, J. (2017). Salud mental positiva. In V. Fragoso \& M. Sotto Mayor (Eds). Gerontologia e Transdisciplinaridade I (pp. 121-139). São Paulo: Editora do Portal do Envelhecimento, Portal Edições. 
Marôco, J., Marôco, A. L., Leite, E., Bastos, C., Vazão, M. J., \& Campos, J. (2016). Burnout em profissionais da saúde Portugueses: Uma análise a nível nacional. Acta Médica Portuguesa, 29(1), 24-30. Doi:10.20344/ amp6460

Marques-Pinto, A. \& Chambel, M.J. (2008). Burnout e engagement em contexto organizacional: estudos com amostras portuguesas. Lisboa: Livros Horizonte.

Marques-Pinto A., Jesus E. H., Mendes A. M. O. C., \& Fronteira I. S. E. (2015). Estudo RN4Cast em Portugal: Work Engagement dos enfermeiros. Revista Investigação em Enfermagem, 10(2), 26-37. Disponível em: https://run.unl.pt/handle/10362/36965

Marques-Pinto, A., Jesus, É. H., Mendes, A. M. O. C., Fronteira, I., \& Roberto, M. S. (2018). Nurses' intention to leave the organization: A mediation study of professional burnout and engagement. The Spanish Journal of Psychology, 21, e32, 1-10.Doi:10.1017/sjp.2018.30

Maslach, C., Schaufeli, W. B., \& Leiter, M. P. (2001). Job burnout. Annual Review of Psychology, 52(1), 397-422. Doi:10.1146/annurev.psych.52.1.397
Purvanova, R. K., \& Muros, J. P. (2010). Gender differences in burnout: A meta-analysis. Journal of Vocational Behavior, 77(2), 168-185. Doi:10.1016/j. jvb.2010.04.006

Salvagioni, D. A. J., Melanda, F. N., Mesas, A. E., González, A. D., Gabani, F. L., \& De Andrade, S. M. (2017). Physical, psychological and occupational consequences of job burnout: A systematic review of prospective studies. PLoS ONE, 12(10), 1-29. Doi: 10.1371/ journal.pone.0185781

Schaufeli, W., \& De Witte, H. (2017). Work engagement in contrast to burnout: Real or redundant? Burnout Research, 5, 1-2. Doi: 10.1016/j.burn.2017.06.001

Shah, D. (2009). Healthy worker effect phenomenon. Indian Journal of Occupational and Environmental Medicine, 13(2), 77-79. Doi: 10.4103/0019-5278.55123

Watanabe, M., \& Yamauchi, K. (2018). The effect of quality of overtime work on nurses' mental health and work engagement. Journal of Nursing Management, 26(6), 679-688. Doi:10.1111/jonm.12595 